\title{
Study on Site-specific Polishing of Polycrystalline Diamond Film by KrF Excimer Laser
}

\author{
Lanxi Wang*, Xuekang Chen, Gan Wu, Shengzhu Cao, Yuqing Xiong, Jinxiao Wang, Dongcai Zhao \\ "National Key Laboratory of Science and Technology on Vacuum Technology and Physics, Lanzhou \\ Institute of physics, P.O. Box 94, Lanzhou 730000, China \\ E-mail :wanglanxi@live.com
}

\begin{abstract}
A self-standing diamond film with thickness of $250 \mu \mathrm{m}$ and root mean square roughness of $3.314 \mu \mathrm{m}$ was polished by a $248 \mathrm{~nm}$ pulsed excimer laser in site-specific mode. The influences of laser incidence angle and energy fluence on polishing performances were studied. The roughness of polished diamond surface was found to decrease first and then increase with laser incidence angle decreasing when fixed the laser energy and laser pulse number. It can be concluded that decreasing the laser incidence angle behaved as reducing the projected area or increasing the energy fluence on the diamond film surface, which enhances the polishing rate. The polishing rate increases logarithmically with the increase of energy fluence at the same laser incidence angle and laser pulse number, from which the polishing threshold of $0.44 \mathrm{~J} \cdot \mathrm{cm}^{-2}$ can be deduced. Additionally, the Raman spectra of pristine and polished diamond films confirm that a residual graphite layer was preserved after laser polishing.
\end{abstract}

DOI: $10.2961 /$ jlmn.2017.02.0002

Keywords: Diamond film; Pulsed excimer laser; Polishing; Surface roughness; Energy fluence threshold

\section{Introduction}

Up to now, diamond films prepared by chemical vapor deposition (CVD) on heterogeneous substrates like silicon, quartz and so on are mostly polycrystalline with enhanced [111] orientation. This kind of diamond film is featured as coarse grain and rough surface with roughness of up to dozens of microns, which becomes a barrier in the way of diamond applications in electronics, optics, thermotics, tribology and so on [1,2]. Thus, polishing diamond becomes a key technology for practical. At present, the surface of polycrystalline diamond films can be polished to be very smooth by using traditional contacting polishing methods such as thermochemical polishing, chemical mechanical polishing and so on [2,3]. However, crack or impurity introduction usually happens to polycrystalline diamond films during contacting polishing [4-8]. In contrast, laser polishing, as one of the non-contacting polishing methods, possesses several advantages of low damage rate, local and scanning polishing ability, no special demand for sample shape, high polishing rate, etc $[9,10]$. Laser polishing is now becoming one of the fastest developing and industrializing diamond polishing methods.

In the process of laser polishing diamond film, parameters of laser wavelength [11], laser incidence angle [12], polishing atmospheres [13] and so on often affect the polishing results. For achieving good polishing results, the polishing mechanism and some technological parameters should be understood well. So, in this work, a pulsed excimer laser with wavelength of $248 \mathrm{~nm}$ and pulse width of 25 ns was used for polishing a free standing polycrystalline diamond film in site-specific mode. It was designed to get some technological rules and parameters of $\mathrm{KrF}$ excimer laser polishing polycrystalline diamond film instead of achieving the minimum roughness. The results are expected to be used as references for further diamond fine polishing.

\section{Experimental}

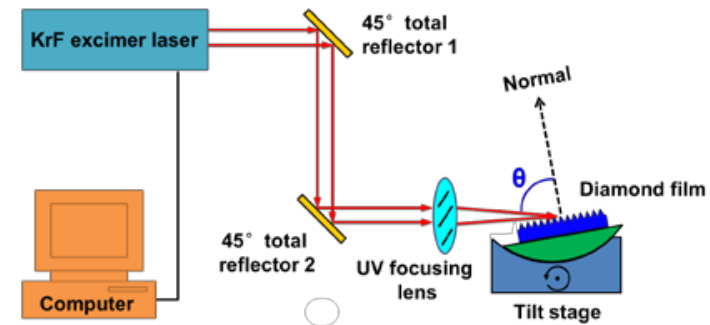

Fig. 1 The schematic of laser polishing setup

A $\mathrm{KrF}$ excimer laser with wavelength of $248 \mathrm{~nm}$ and pulse width of 25 ns was used for polishing a free standing polycrystalline diamond film. The diamond film was deposited on a Si (100) substrate in a microwave chemical vapor deposition chamber by using a gas mixture of hydrogen and methane $\left(\mathrm{CH}_{4}: 6 \mathrm{sccm}+\mathrm{H}_{2}: 600 \mathrm{sccm}\right)$ at $950{ }^{\circ} \mathrm{C}$. The Si substrate was removed in a $60 \% \mathrm{KOH}$ solution at $100^{\circ} \mathrm{C}$. The thickness and Root Mean Square (RMS) roughness of the polycrystalline diamond film are $250 \mu \mathrm{m}$ and $3.314 \mu \mathrm{m}$, respectively. Fig.1 shows the schematic of the laser polishing setup in this work. Reflector 1 and 2 are $248 \mathrm{~nm}$ ultraviolet total reflectors with reflection angle of $45^{\circ}$. Then, the laser was focused to be a $1 \times 3 \mathrm{~mm}$ rectangle spot by a quartz lens. The diamond film was placed on a tilt stage which was used for changing the incidence angle $\theta$. $\theta$ is defined as the angle 
between the focused laser beam and the diamond film normal. Laser repetition frequency and laser energy can be changed by the control computer.

Film roughness was measured by MicroXAM-3D surface profiler whose highlights are non-contact, no stylus and ultra-precise surface height sensitivity. The surface topography of diamond films was observed by JEOL JSM-5600LV Scanning Electron Microscope (SEM) in which diamond films were tilted $45^{\circ}$ up on the sample stage. Crystalline quality of the diamond films before and after polishing was investigated by a mico-confocal Raman spectrometer Horiba LabRAM HR Evolution. The 325nm laser line from a He-Cd laser was used as the exciting source.

\section{Results and discussion}

The diamond films were polished by the laser with repetition frequency of $20 \mathrm{~Hz}$, laser energy of $400 \mathrm{~mJ}$, pulse number of 4000 and different laser incidence angles. Fig. 2 shows the relationship between RMS roughness of the diamond films and laser incidence angles. The RMS roughness of the diamond films decreases to a minimum value and then increases with the decrease of laser incidence angle. Fig.3(a) to (e) are surface topographies of the diamond films polished with different laser incidence angles. The projective area of the laser beam on diamond film decreases equivalently when the laser incidence angle decreases. Consequently, laser energy fluence increases significantly. Thus, the laser ablation increases with the decrease of laser incidence angle. The optimal laser incidence angle drops between $75^{\circ}$ and $80^{\circ}$. There are plenty of pits between crystalline grains in polycrystalline diamond film. Ablated tails are usually produced from the edge of pits by grazing-incidence laser [12], which can be seen in Fig.3(c). As the laser incidence angle decreases more, laser ablated the tails much deeper. Then, the polished area often presents corrugate topography which is shown in Fig.3(f). The RMS roughness, in turn, increases with the decrease of laser incidence angle.

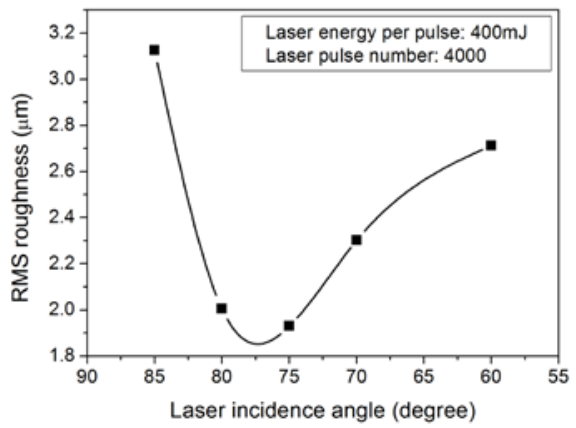

Fig. 2 The relationship between root mean square roughness of the diamond films and laser incidence angles
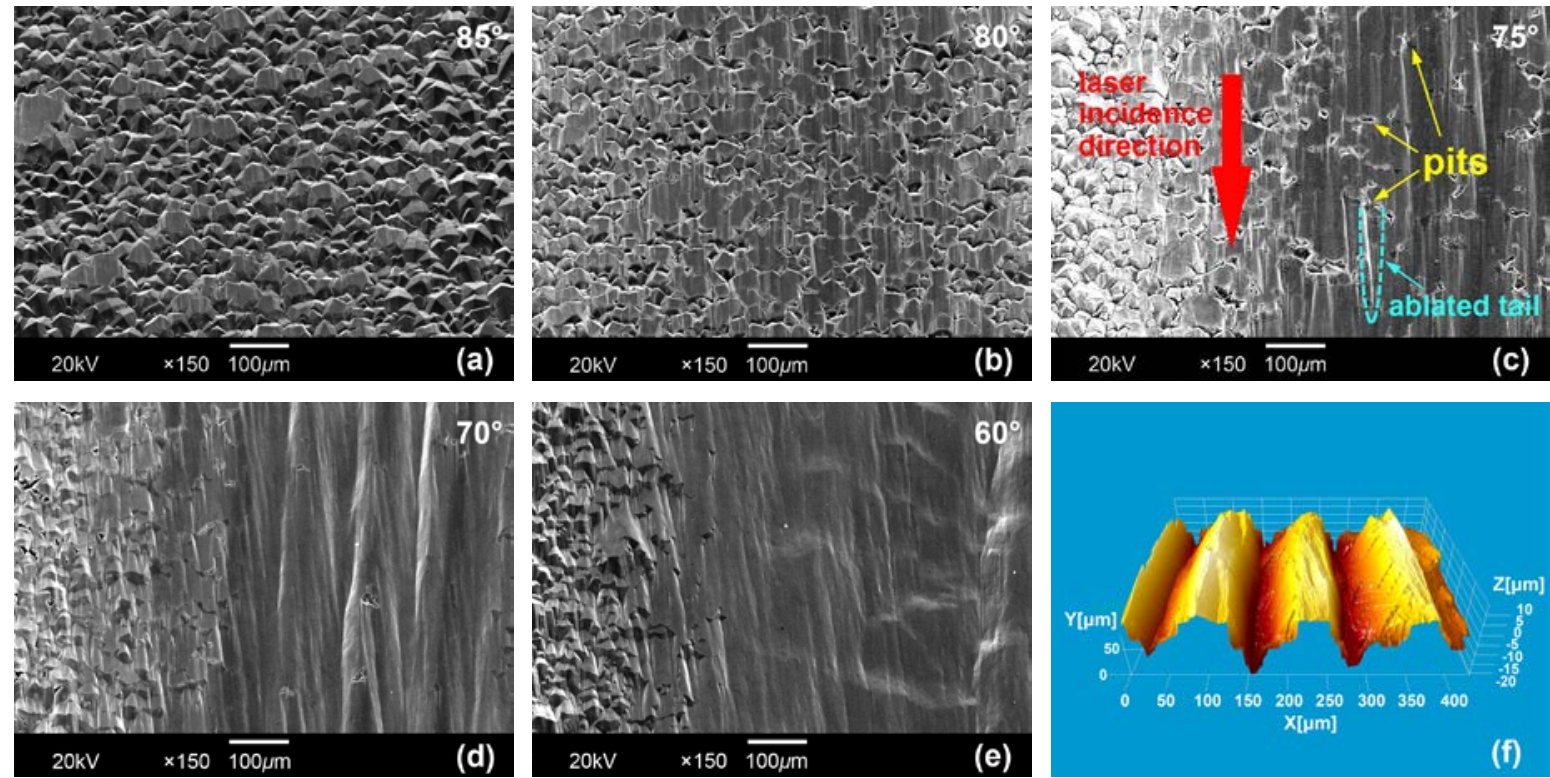

Fig. 3 (a)-(e) are surface topographies of the diamond films polished with different laser incidence angles,

(f) is the $3 \mathrm{D}$ profile of the diamond film polished with the $70^{\circ}$ laser incidence angle.

Table 1 The RMS roughness of polished diamond films using different laser energy fluence

\begin{tabular}{ccc}
\hline $\begin{array}{c}\text { Laser energy } \\
\text { per pulse }(\mathbf{m J})\end{array}$ & $\begin{array}{c}\text { Laser energy } \\
\text { fluence }\left(\mathbf{J} \cdot \mathbf{c m}^{-\mathbf{2}}\right)\end{array}$ & $\begin{array}{c}\text { RMS roughness } \\
(\boldsymbol{\mu m})\end{array}$ \\
\hline 100 & 0.58 & 2.869 \\
200 & 1.16 & 2.742 \\
300 & 1.74 & 1.932 \\
400 & 2.32 & 1.652 \\
500 & 2.90 & 1.447 \\
600 & 3.48 & 1.299 \\
\hline
\end{tabular}

Table 1 lists the variation of RMS roughness of the polished diamond films with laser energy fluence by fixing laser incidence angle of $80^{\circ}$, laser repeat frequency of $20 \mathrm{~Hz}$ and laser pulse number of 6000.

The RMS roughness of diamond film decreases with the increase of laser energy fluence. The laser polishing rate per pulse can be expressed here by:

$$
V=\left(R_{i}-R_{p}\right) / N
$$

Here, $R_{i}$ is the RMS roughness of as-prepared diamond film, $R_{p}$ is the RMS roughness of polished diamond film, $N$ is the laser pulse number. Laser polishing rate per pulse 
increases with laser energy fluence, which is shown in Fig.4. The polishing rate can exceed $3.0 \times 10^{-4} \mu \mathrm{m}$ per laser pulse when laser energy fluence is greater than $3.48 \mathrm{~J} \cdot \mathrm{cm}^{-2}$.

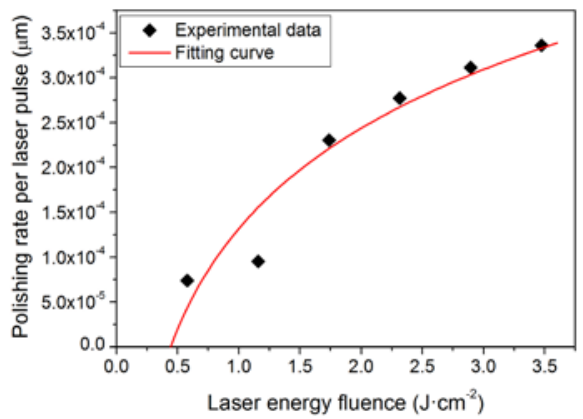

Fig.4 The relationship between laser polishing rate and laser energy fluence

Diamond is a wide band gap semiconductor with band gap width of $5.5 \mathrm{eV}$ which is higher than the photon energy of the 248nm excimer laser. Laser absorption in diamond follows the equation as follows [14]:

$$
E_{z}=E_{0}(1-R) e^{-\alpha z}
$$

$E_{z}$ is the laser energy fluence at depth $z$ penetrating into the diamond film. $E_{0}$ is the incident laser energy fluence. $R$ is the reflection coefficient of the laser on diamond film surface. $\alpha$ is the absorption coefficient of the laser in the diamond film, which is about $1 \times 10^{3} \mathrm{~cm}^{-1}$ [15]. There should be a threshold energy fluence $E_{t}$ for diamond to be ablated by the laser. If $E_{z}$ equals to $E_{t}$, then the diamond within $z$ penetration depth will be ablated. So, $z$ can be drawn:

$$
z=(1 / \alpha) \operatorname{Ln}\left[E_{0}(1-R) / E_{t}\right]
$$

The schematic of laser grazing incidence on diamond film surface is shown in Fig.5. In this situation, the laser can scrape the pyramidal diamond grain tips instead of drilling the diamond film too much. According to ref. [12], the final amplitude $h$ of diamond pyramid grain after polishing under grazing incidence condition obeys the following equation:

$$
h(I)=H \tan \phi_{0}(I) / \tan \phi_{1}
$$

Here, $I$ is the laser intensity, $H$ is the initial amplitude of diamond grain. Under the condition of approximation, roughness of pristine diamond and polished diamond can be expressed by,

$$
\begin{aligned}
& R_{\mathrm{i}} \sim H \\
& R_{\mathrm{p}} \sim h
\end{aligned}
$$

So, the polishing rate can be deduced by

$$
V \sim H-h
$$

The penetrate depth of laser in diamond $z$ has a certain geometric relationships with $H$ and $h$, so

$$
V \sim z f\left(\phi_{0}, \phi_{1}\right)
$$

When the laser intensity $I$ and laser incidence angle are fixed, $f\left(\phi_{0}, \phi_{1}\right)$ should be a constant. Thus, it can be considered that laser polishing rate $V$ is proportional to $z$,

$$
V=A z=B \operatorname{Ln}\left(C E_{0}\right)
$$

Here, A, B and C are constants. However, it is a rough approximation. But experiment results indeed follow the logarithmic rules under grazing incidence, which were also reported by other research groups [16]. The solid line in Fig.4 is the curve fitted by Eq.(9). The threshold energy fluence without considering laser reflection is $0.44 \mathrm{~J} \cdot \mathrm{cm}^{-2}$ which can be achieved from the intersection point of the fitting curve and $x$ axis. According to the energy of $s p^{3} \mathrm{C}-\mathrm{C}$ bond in diamond, the theoretical threshold energy fluence can be calculated to be $0.37 \mathrm{~J} \cdot \mathrm{cm}^{-2}$ [17] which is most probably larger than the real value for CVD diamond film because of grain boundaries, defects, impurities and so on. The experimental threshold energy fluence in this work is consistent with the theoretical value by considering the laser reflection.

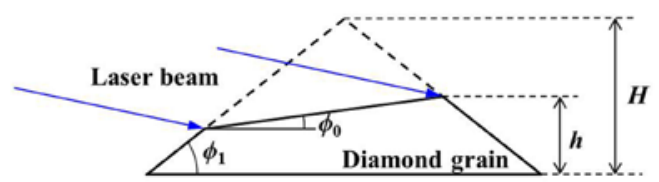

Fig.5 The schematic of laser grazing incidence on diamond film
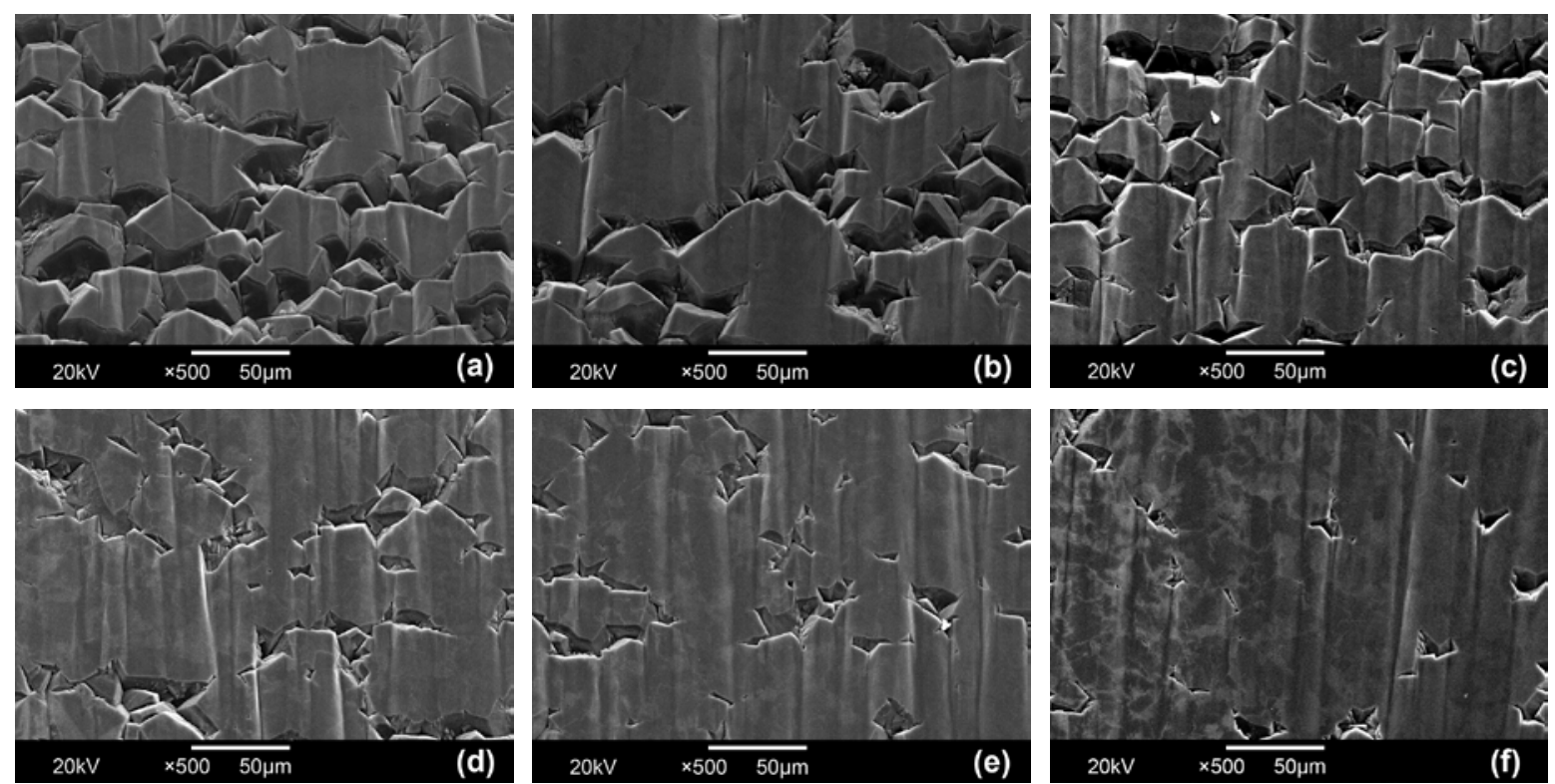

Fig.6 Surface topographies of the diamond films polished under different laser energy fluences:

(a) $0.58 \mathrm{~J} \cdot \mathrm{cm}^{-2}$, (b) $1.16 \mathrm{~J} \cdot \mathrm{cm}^{-2}$, (c) $1.74 \mathrm{~J} \cdot \mathrm{cm}^{-2}$, (d) $2.32 \mathrm{~J} \cdot \mathrm{cm}^{-2}$, (e) $2.90 \mathrm{~J} \cdot \mathrm{cm}^{-2}$, (f) $3.48 \mathrm{~J} \cdot \mathrm{cm}^{-2}$ 
A process of diamond transforming into some material which has strong laser absorption and low thermal conductivity should be achieved when the diamond is being polished by the laser [18]. Some research achievements have indicated that the threshold energy fluence required for ablating CVD diamond film and graphite was nearly the same by using a $\mathrm{KrF}$ excimer laser with wavelength of $248 \mathrm{~nm}$ and pulse width of $23 \mathrm{~ns}$ [19]. The process of ablating diamond is known as a graphitization of diamond, in which the diamond is removed via graphitization and graphite sublimation. Predictably, diamond can be removed more easily by absorbing more energy with the increase of laser energy fluence. Fig.6(a)-(f) are topographies of the diamond films polished by different laser energy fluence. Obviously, the polished area becomes smoother by increasing laser energy fluence. But laser polishing rate trends to increase slowly as shown in Fig.4.

As shown in Fig.7, Raman spectra of pristine and polished diamond films were measured to check the influence of laser polishing on diamond crystalline quality. The Raman spectrum of pristine diamond film has a strong diamond peak at $1330.49 \mathrm{~cm}^{-1}$ with width at half maximum (FWHM) of $12.08 \mathrm{~cm}^{-1}$ in Fig.7(a). Meanwhile, a weak peak is observed to be centered at $1578.09 \mathrm{~cm}^{-1}$, which indicates the presence of graphite phase in diamond film. Graphite is usually inclined to remain in some interspace between coarse diamond grains. After polishing, the D band centered at $1399.69 \mathrm{~cm}^{-1}$ and the $\mathrm{G}$ band centered at $1568.99 \mathrm{~cm}^{-1}$ appear in the Raman spectrum as shown in Fig.7(b), which are usually assigned to microcrystalline graphite and graphite respectively. Similar results have been also reported by other research groups [20-22]. Diamond will experience a series of processes by laser ablation such as heat-absorption, vaporization, graphitization, etc. As a consequence, a residual graphite layer transited from diamond is preserved [23]. Nevertheless, the residual graphite layer can be removed easily by atomic hydrogen for further applications [24].

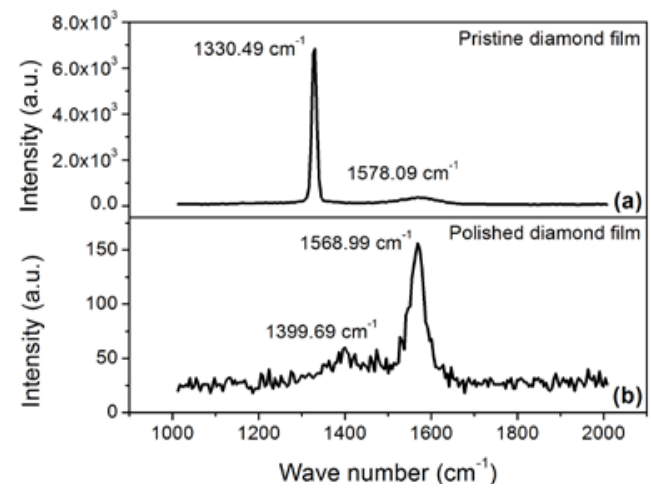

Fig.7 The Raman spectrum of (a) the pristine and (b) polished diamond film

\section{Conclusion}

It is efficient for 248nm ultraviolet pulsed excimer laser to polish polycrystalline diamond film according to the results in this work. Polycrystalline diamond film with energy threshold of only $0.44 \mathrm{~J} \cdot \mathrm{cm}^{-2}$ could be polished easily by the laser. The polishing rate can exceed $3.0 \times 10^{-4}$ $\mu \mathrm{m}$ per laser pulse. Some points should be considered for the engineering application of $\mathrm{KrF}$ excimer laser in polishing polycrystalline diamond film:

1. Laser incidence angle may be controlled between $75^{\circ}-80^{\circ}$. Too large incidence angle leads to low polishing rate. Too small usually causes diamond film corrugate topography which enhances the roughness. Practically, it is necessary for achieving large area smooth diamond film by rotating the sample or scanning the laser beam.

2. The polishing rate shows a logarithmic increase with laser energy fluence. It is useful for increasing polishing rate by moderately raising laser energy fluence. However, it should be a compromise between polishing rate and laser consumptions.

3. A residual graphite layer was found to be preserved on the polished surface. But it is not difficult to remove the residual graphite layer and restore the fresh diamond surface by atomic hydrogen for further applications.

\section{Acknowledgement}

This work was financially supported by the National Natural Science Foundation of China (Grant No NSFC51135005).

\section{References:}

[1] R.S. Sussmann: "CVD Diamond for Electronic Devices and Sensors", (John Wiley \& Sons, Chichester, 2009) p.404.

[2] T. Schuelke, T.A. Grotjohn: Diam. Relat. Mater., 32, (2013) 17.

[3] A.P. Malshe, B.S. Park, W.D. Brown, H.A. Naseem: Diam. Relat. Mater., 8, (1999) 1198.

[4] N. Tatsumi, K. Harano, T. Ito, H. Sumiya: Diam. Relat. Mater., 63, (2016) 80.

[5] S.T. Huang, L. Zhou, L.F. Xu, K.R. Jiao: Diam. Relat. Mater., 19, (2010) 1316.

[6] C.Y. Wang, F.L. Zhang, T.C. Kuang, C.L. Chen: Thin Solid Films, 496, (2006) 698.

[7] E.L.H. Thomas, G.W. Nelson, S. Mandal, J.S. Foord, O.A. Williams: Carbon, 68, (2014) 473.

[8] Z. Yuan, Z. Jin, Y. Zhang, Q. Wen: J. Manuf. Sci. Eng., 135, (2013) 041006-1.

[9] S.M. Pimenov, V.V. Kononenko, V.G. Ralchenko, V.I. Konov, S. Gloor, W. Lüthy, H.P. Weber, A.V. Khomich: Appl. Phys. A-Mater., 69, (1999) 81.

[10] V.I. Konov: Laser Photonics Rev., 6, (2012) 739.

[11] T.V. Kononenko, V.G. Ralchenko, I.I. Vlasov, S.V. Garnov, V.I. Konov: Diam. Relat. Mater., 7, (1998) 1623.

[12] V.N. Tokarev, J.I.B. Wilson, M.G. Jubber, P. John, D.K. Milne: Diam. Relat. Mater., 4, (1995) 169.

[13] S. Gloor, S.M. Pimenov, E.D. Obraztsova, W. Lüthy, H.P. Weber: Diam. Relat. Mater., 7, (1998) 607.

[14] S.M. Sze, K.K. Ng: "Physics of Semiconductor Devices ( $3^{\text {rd }}$ edition)", (John Wiley \& Sons, New Jersey, 2006) p.53.

[15] T. Sharda, M.M. Rahaman, Y. Nukaya, T. Soga, T. Jimbo, M. Umeno: Diam. Relat. Mater., 10, (2001) 561. 
[16] A.M. Ozkan, A.P. Malshe, W.D. Brown: Diam. Relat. Mater., 6 (1997) 1789.

[17] R. Windholz, P.A. Molian: J. Mater. Sci., 33, (1998) 523.

[18] M.D. Shirk, P.A. Molian, A.P. Malshe: J. Laser Appl., 10, (1998) 64.

[19] R. Windholz, P.A. Molian: J. Mater. Sci., 32, (1997) 4295.

[20] S.M. Pimenov, G.A. Shafeev, V.I. Konov, E.N. Loubnin: Diam. Relat. Mater., 5, (1996) 1042.

[21] S. Gloor, S.M. Pimenov, E.D. Obraztsova, W. Lüthy, H.P. Weber: Diam. Relat. Mater., 7, (1998)
607.

[22] M. De Feudis, A.P. Caricato, G. Chiodini, M. Martino, E. Alemanno, G. Maruccio, A.G. Monteduro, P.M. Ossi, R. Perrino, S. Spagnolo: Diam. Relat. Mater., 65, (2016) 137.

[23] P.W. Butler-Smith, D.A. Axinte, M. Pacella, M.W. Fay: J. Mater. Process. Technol., 213, (2013) 194.

[24] P. Ascarelli, E. Cappelli, S. Orlando, F. Pinzari: Appl. Surf. Sci., 127-129, (1998) 837.

(Received: September 3, 2016, Accepted: May 15, 2017) 